\title{
Architecture et interdisciplinarité. Le cas d'étude des instituts de la complexité
}

\author{
Fabrizio Li Vigni ${ }^{\mathrm{a}, *}$ \\ Sociologie du numérique, Sciences Po Saint-Germain-en-Laye, Saint-Germain-en-Laye, France
}

\begin{abstract}
Résumé - Un certain nombre d'historiens et de sociologues ont interrogé la relation entre socialisation et architecture. Mais rares sont les auteurs ayant réfléchi au lien entre architecture et production des savoirs scientifiques. Dans cet article, nous présentons une piste de recherche pour approfondir le rapport entre architecture et production de savoirs dits interdisciplinaires. Nous proposons d'enquêter sur les discours, les stratégies et les éléments matériels que les chercheurs déploient pour mettre en place des collaborations scientifiques entre domaines différents. Les cas d'étude sont deux «instituts de la complexité » : le Santa Fe Institute (SFI) et l'Institut des systèmes complexes - Paris Île-de-France (ISC-PIF). Le matériau empirique se fonde sur des entretiens, des ethnographies et des archives.
\end{abstract}

Mots clés : recherche / dispositifs institutionnels / sociologie des sciences / sciences des systèmes complexes / États-Unis / France

\begin{abstract}
Architecture and interdisciplinarity. The case study of complexity institutes. A number of historians and sociologists have questioned the relation between socialization and architecture. Yet the relation between the latter and the production of scientific knowledge has been seldom addressed. In this article, we present a research avenue that aims to explore in greater depth the relation between architecture and the production of so-called interdisciplinary scientific knowledge. We propose to investigate the discourses, strategies and material elements that researchers deploy to set up scientific collaborations between different domains. Our case study is represented by two "complexity institutes": the Santa Fe Institute and the Complex Systems Institute of Paris Ile-de-France. Empirical material consists of interviews, ethnographies and archives. We ask a series of questions: how is the interdisciplinary use of architecture discussed and reported? How is it materially established? What are the observable research practices in these architectural spaces?
\end{abstract}

Keywords: research / institutional devices / sociology of sciences / complex systems sciences / USA / France

Un certain nombre d'historiens et de sociologues ont interrogé la relation entre socialisation et architecture (Winner, 1980; Bourdieu, 1980). Mais rares sont les auteurs ayant réfléchi au lien entre architecture et production des savoirs scientifiques. Dans un livre collectif intitulé The architecture of science, l'historien des sciences Peter Galison (1999) se demande comment les bâtiments de recherche configurent l'identité des chercheurs, mais

\footnotetext{
*Auteur correspondant : livignifabrizio@gmail.com

${ }^{a}$ F. Li Vigni est enseignant-chercheur contractuel à Sciences Po Saint-Germain-en-Laye et associé au Laboratoire interdisciplinaire sciences innovations sociétés (Université Gustave-Eiffel, Champs-sur-Marne) et au Groupe de sociologie pragmatique et réflexive (École des hautes études en sciences sociales, Paris).
}

aussi comment les sciences que ces derniers pratiquent structurent l'architecture dans laquelle ils viennent travailler. La thèse du livre est claire : l'architecture fait partie de la culture, au sens où elle incorpore et à la fois transmet des éléments pratiques, épistémiques, normatifs, ontologiques, etc.

Dans cet article, nous voudrions exposer une piste de recherche pour approfondir le rapport entre architecture et production de savoirs dits interdisciplinaires ${ }^{1}$. Par la présentation de deux cas d'étude, nous proposons

\footnotetext{
${ }^{1}$ Cette recherche est issue d'un travail doctoral d'ordre historique et sociologique sur le domaine des «sciences des systèmes complexes », avec une focale franco-européenne et états-unienne (Li Vigni, 2018).
} 
d'enquêter sur les discours, les stratégies et les éléments matériels que les chercheurs déploient pour mettre en place des collaborations scientifiques entre domaines différents. Comment l'usage de l'architecture à des fins interdisciplinaires est-il discuté et annoncé? Comment est-il matériellement mis en place? Quelles sont les pratiques de recherche observables dans ces espaces architecturaux? Où et de quelle manière s'engendre l'interdisciplinarité ? Quelle est l'histoire même de l'usage de l'architecture à des fins de science interdisciplinaire?

L'un des aspects intéressants des «sciences des systèmes complexes » - l'objet d'étude de notre doctorat (Li Vigni, 2018 ${ }^{2}$ - réside dans le fait que les scientifiques s'en réclamant mènent une réflexion explicite et militante sur l'usage architectural de leurs instituts. Dans ce travail exploratoire, nous donnerons quelques éléments de réponse à la série de questions listées ci-dessus. Mais il faut rappeler que les instituts de la complexité sont loin d'être les premiers ou les seuls à avoir posé la question ouvertement. Dans ses études sur le Projet Manhattan, par exemple, Galison (1997) avait déjà mis en lumière les réflexions de scientifiques, politiciens et ingénieurs sur la mise en place d'un laboratoire qui puisse prendre en compte structurellement les collaborations entre experts de domaines différents. D'ailleurs, les centres de recherche qui aujourd'hui se revendiquent interdisciplinaires, à la fois dans leur composition sociale et dans leur architecture, sont légion dans tous les pays et touchent plusieurs domaines de recherche. En revanche, peu d'enquêtes ont été menées sur ces espaces et sur leurs pratiques.

En dépassant le réductionnisme du déterminisme social et du déterminisme technologique (Wyatt, 2008), il s'agit ici de s'interroger sur la dynamique de ce qu'on propose d'appeler l'ingénierie de l'interdisciplinarité - à savoir l'ensemble des stratégies discursives, organisationnelles et architecturales qui sont mises en place par des collectifs de recherche dans l'objectif de faire naître et d'entretenir un type de travail scientifique dit interdisciplinaire.

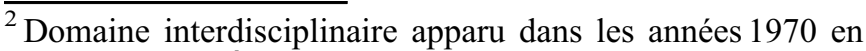
Europe et aux États-Unis et ayant accédé à une dimension internationale via le Santa Fe Institute dans les années 1980, les «sciences de la complexité» regroupent un ensemble de sciences naturelles, sociales et de l'ingénieur se proposant d'étudier les «systèmes complexes », c'est-à-dire des ensembles composés de nombreux éléments en interaction réciproque et donnant lieu à des propriétés émergentes (ex. : réseaux génétiques, écosystèmes, villes, marchés financiers, épidémies, etc.). Les scientifiques de ce domaine se proposent de les étudier avec des outils informatiques, mathématiques et physiques, comme la théorie des réseaux, les modèles à base d'agents et les systèmes multi-agents, les automates cellulaires ou la physique statistique.
}

La première partie prend pour étude le Santa $\mathrm{Fe}$ Institute (SFI), un visiting institute privé situé au Nouveau-Mexique, fondé en 1984. La deuxième partie se focalise sur l'Institut des systèmes complexes - Paris Île-de-France (ISC-PIF), fondé sous la forme d'un Groupement d'intérêt scientifique en 2005, puis devenu une Unité de service et de recherche du CNRS en 2013, puis une Unité d'appui à la recherche depuis 2021. Le premier de ces deux centres est célèbre dans le monde entier pour avoir lancé l'expression «science de la complexité » ainsi que la notion de «système complexe adaptatif» (Waldrop, 1992; Helmreich, 1998). Le second fait partie d'un réseau franco-européen concrétisé dans le Réseau national des systèmes complexes (RNSC) et dans la Société européenne des systèmes complexes, et se veut un héritier du SFI (CNRS et al., 2005 ; Bourgine et al., 2009; Bourgine et Johnson, 2006).

Trois dizaines d'entretiens, une dizaine de courtes ethnographies et quelques dizaines de pages d'archives représentent le matériau empirique sur lequel s'appuie cette réflexion.

\section{Le cas d'étude du SFI}

Depuis le début de son histoire, le SFI a toujours mis en avant le rôle de l'architecture pour encourager une certaine manière de faire de la science. Comme le disait le premier vice-président de l'institut, Mike Simmons, dans un entretien publié dans un «bulletin» de 1990, la future structure du centre aurait dû être «pensée soigneusement» pour devenir "presque invisible» afin de favoriser les interactions (SFI, 1990, p. $10^{3}$ ). L'architecture était envisagée comme un élément pouvant multiplier les idées et les échanges, la créativité et la productivité des membres de l'institut.

\section{Les intérêts pluridisciplinaires des premiers membres}

Si l'on prend en compte le parcours des fondateurs du SFI, cet intérêt pour l'interdisciplinarité et pour l'architecture devant l'accueillir n'est pas étonnant: ces fondateurs sont des scientifiques ayant travaillé dans l'un des rares laboratoires américains, le Los Alamos National Laboratory (LANL), où il est possible de réaliser la totalité d'un processus technoscientifique, de la recherche dite fondamentale jusqu'à la construction concrète de dispositifs technologiques. Dans un bulletin du SFI de 2004, celui qui fut le premier président de l'institut, George Cowan, dit avoir appris au LANL les

\footnotetext{
${ }^{3}$ Les bulletins sont une publication annuelle ou bisannuelle que le SFI a envoyée à ses membres et à ses donateurs de 1986 à 2014, pour présenter les recherches en cours et pour communiquer sur les évolutions en interne.
} 
méthodes de «social engineering» du travail interdisciplinaire, qui consistent dans l'organisation de «marriage $(s)$ » entre représentants de spécialités différentes à travers l'attrait pour la réalisation d' "exciting things » (SFI, 2004, p. 3-4). D'autres institutions avaient inspiré les fondateurs du SFI, notamment l'Aspen Center for Physics, l'Institute of Advanced Studies de Princeton et le Jonas Salk Institute, qui sont tous des centres sans barrières disciplinaires et administratives (SFI, 1990). En outre, ces fondateurs considéraient le Projet Manhattan, les simulations Monte-Carlo, la biologie moléculaire, l'intelligence artificielle et les sciences cognitives comme des modèles des "synthèses émergentes » (SFI, 1995-1996; Pines, 1988 ; SFI, 1987 ; SFI, 1984 ; SFI, 1986; Cowan, 2010).

Enfin, dans plusieurs textes d'archives et publics, les fondateurs se présentaient comme des hommes de la Renaissance ${ }^{4}$. Par le biais de plusieurs documents de promotion de l'institut (entretiens, articles et livres grand public), ils créèrent une auto-mythologie dans laquelle ils se targuaient d'un intérêt pour de multiples disciplines (SFI, 1989). Murray Gell-Mann, par exemple, énumérait les siennes: physique théorique (son domaine de recherche), ornithologie, linguistique, archéologie, histoire, psychologie, géopolitique et environnement (SFI, 1989, p. 11). Plus généralement, tous les membres du SFI ont toujours eu dès le départ un intérêt pour au moins deux disciplines.

\section{La planification de l'interdisciplinarité}

Le SFI est situé au 1399 Hyde Park Road à Santa Fe. Mais il n'a pas toujours été au même endroit. Les fondateurs n'avaient tout d'abord qu'une boîte postale, puis ils installèrent l'institut dans un couvent, ensuite dans un bâtiment de bureaux commerciaux et enfin sur les collines résidentielles où il se trouve aujourd'hui.

Lorsque le SFI fit son quatrième et dernier déménagement, la direction interpella les scientifiques, les gestionnaires et les employés pour connaître quelles étaient les caractéristiques qu'ils aimeraient trouver dans ce qui est appelé de nos jours le Cowan Campus (en honneur du premier président, qui avait fait la donation la plus importante pour acquérir la villa).

L'expérience dans le couvent avait marqué les esprits des fondateurs et des premiers membres du SFI : selon leurs témoignages, le petit espace induisait des contacts informels favorisant les collaborations interdisciplinaires. Dans les intentions de la direction, le Cowan Campus devait conserver le même esprit que le couvent, tout en fournissant des espaces plus aptes à accueillir une vie

\footnotetext{
${ }^{4}$ Aucune femme n'était présente dans les premières réunions des fondateurs du SFI.
}

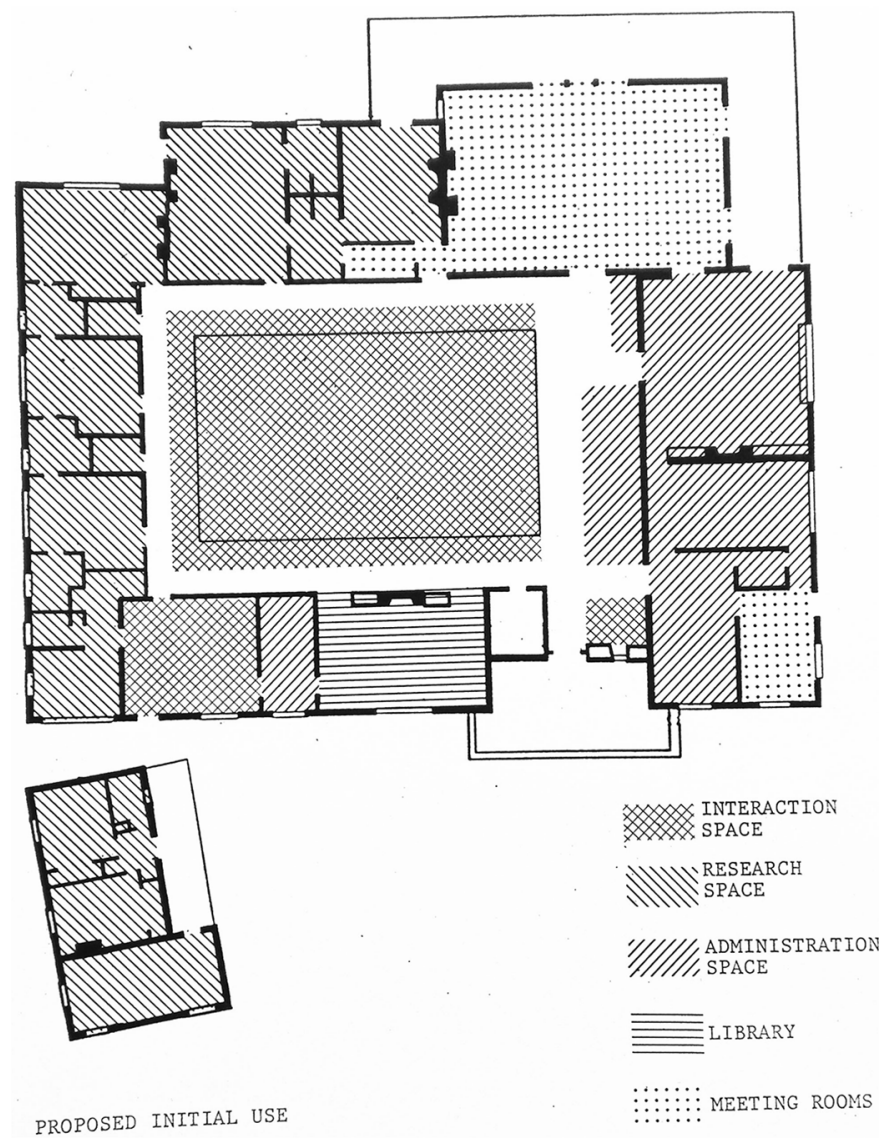

Fig. 1. Plan du Cowan Campus (source: SFI, 1993).

sociale qui, entre-temps, s'était accrue - le nombre de chercheurs s'étant élevé de vingt à plus de cent- (SFI, 1993). Le bulletin du SFI de l'hiver 1996-1997 présentait la nouvelle structure en mettant en lumière sa taille plus grande et sa fonction d'encouragement de la collaboration entre représentants de sciences différentes (SFI, 1996-1997, p. 24). Mais qu'entendait la direction de l'institut par encourager la collaboration scientifique? La Fig. 1 montre que l'élément architectural fondamental est la présence de bureaux petits et partagés, couplés à des grands espaces communs censés faciliter les échanges. En particulier, les plans définitifs du SFI s'inspirent de la méthode "caves and commons», inventée et appliquée au MIT par le cognitiviste Marvin Minsky dans les années 1960 et aujourd'hui en vogue dans le monde des entreprises, notamment informatiques (Brand, 1995). Dans cette logique, les espaces communs doivent être confortables et bien équipés pour encourager les résidents à y rester. Les différents étages du SFI comprennent en effet de grands tableaux, de grands canapés, des tables de travail et plusieurs bibliothèques. Les murs qui séparent l'interne de l'externe de la structure, mais aussi les murs des bureaux, sont en grande majorité vitrés, le but étant de créer un contact visuel entre les scientifiques dans les bureaux et les passants, 
mais aussi de permettre à quiconque d'écrire des équations et des modèles où qu'il soit dans le bâtiment (SFI, 1996-1997, p. 24-25).

Un rapport interne de l'institut mentionne les différentes positions qui se sont manifestées lors des entretiens que la direction a conduits pour dresser les plans du Cowan Campus (SFI, 1993). Les différends concernaient toutes sortes d'aspects pratiques : combien de personnes par bureau; faut-il séparer le staff des scientifiques; faut-il créer une ambiance silencieuse ou faut-il faciliter le contact même bruyant, etc. D'après le conseil d'administration de l'institut, l'isolement du campus au milieu des collines de Santa Fe en faisait un lieu propice à la concentration. Mais à l'intérieur et sur les terrasses, les relations devaient être maximisées. Cette déclaration d'intention fut partiellement concrétisée en excluant un usage individuel des bureaux. Même le nombre des accès à la structure fut pensé à des fins d'interactions, le faible nombre de portes étant censé contraindre les personnes à se rencontrer. La direction de l'institut veilla cependant à séparer les activités bruyantes des activités silencieuses: la salle de conférences, la cuisine et les tables à manger sont en effet concentrées au premier étage.

Pendant notre séjour, seul un nombre restreint d'individus, toujours les mêmes, profitait des grands espaces et des grands tableaux pour discuter longuement. Les autres résidents restaient dans les bureaux, qui étaient le plus souvent occupés par une seule personne à la fois. L'une des bibliothécaires du SFI toutefois a porté notre attention sur un aspect absent des archives : le rôle de la nourriture et du thé dans la socialisation scientifique de l'institut ${ }^{5}$. Les voisins du SFI avaient accepté l'implantation de l'institut dans leur zone, à condition que le repas soit servi en interne. Cela afin d'éviter que les scientifiques prennent leurs voitures tous les jours pour aller déjeuner en ville. Cette contrainte a conduit à intégrer le dispositif gastronomique, ainsi que le thé de l'après-midi, dans les stratégies de l'ingénierie interdisciplinaire du centre de recherche. Les tables à manger et la machine à café ont, ainsi, été délibérément situées au premier étage, pour contraindre les chercheurs à se rencontrer au même endroit deux fois par jour ${ }^{6}$.

\section{Où s'engendre l'interdisciplinarité ?}

Les moments dans la vie du SFI qui nous semblent les plus importants dans la formation de nouveaux projets interdisciplinaires s'appellent "integrative workshops». À mi-chemin du congrès et de l'école d'été, un «atelier intégratif » se distingue de ces deux dispositifs classiques

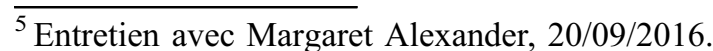

${ }^{6}$ Entretien avec Ronda Butler-Villa, 03/11/2016.
}

par sa longueur - une, voire deux semaines - et par l'ambiance hautement décontractée qui le caractérise. Ces ateliers rassemblent deux à trois dizaines de scientifiques maîtrisant deux, voire plusieurs disciplines différentes, pour qu'ils collaborent dans le but de trouver de nouveaux langages de recherche. Chaque participant intervient en informant les autres de la manière dont on conçoit et traite l'objet d'étude de l'atelier dans sa propre discipline. À la différence des conférences, dans les ateliers du SFI, l'exposé est souvent libre, improvisé et sans limites temporelles strictes, tandis que le conférencier peut être interrompu à tout moment par l'auditoire (SFI, 1996, p. 6). Le dynamisme, le bouillonnement constant, l'anticonformisme créatif sont soulignés et valorisés par le SFI, qui considère l'atelier intégratif comme sa marque de fabrique.

L'un des premiers ateliers organisés par le SFI a réuni des physiciens et des économistes pendant quinze jours, dans le but de produire de nouveaux modèles pour appréhender les marchés financiers : c'était la préoccupation du PDG de Citibank qui avait financé l'atelier (SFI, 1988). Lors de ces rencontres, les prémices de ce qui sera plus tard appelé " économie de la complexité » furent posées $^{7}$ :

«In September 1987, twenty people came together at the Santa Fe Institute to talk about "the economy as an evolving, complex system". Ten were theoretical economists, invited by Kenneth J. Arrow, and ten were physicists, biologists, and computer scientists, invited by Philip W. Anderson. [...] For ten days, economists and natural scientists took turns talking about their respective worlds and methodologies. [...] The meeting left two legacies. The first was a volume of essays, The Economy as an Evolving Complex System, edited by Arrow, Anderson, and David Pines. The other was the founding, in 1988, of the Economics Program at the Santa Fe Institute, the Institute's first resident research program. [...] Looking back over the developments in the past decade and the papers produced by the program, we believe that a coherent perspective sometimes called the "Santa Fe approach" - has emerged within economics ». (Arthur et al., 2015, p. 89-91)

La configuration qui fut celle de cette rencontre entre physiciens et économistes caractérisera la plupart des ateliers intégratifs suivants, qui se suivront au rythme d'une vingtaine par an. À l'exception des ateliers dédiés à la convergence entre différentes théories à l'intérieur d'une même discipline, les ateliers du SFI ont toujours eu comme but de réunir différents spécialistes autour d'un même concept - système complexe, robustesse, contagion, réseau, évolution, etc. - pour parvenir à des convergences théoriques et méthodologiques à travers

\footnotetext{
${ }^{7}$ Approche refusant le postulat néoclassique des marchés en équilibre et décrivant ces derniers comme étant des systèmes autoorganisés, évolutifs et distribués.
} 
des séances de brainstorming. Nous n'observons pas un usage particulier de l'architecture dans une telle entreprise, car ces rencontres se passent dans des salles de lycée à Santa $\mathrm{Fe}$ ou dans la salle de conférences du SFI, sans que cela change le type d'interactions qui y ont lieu. Le choix des participants - rigoureusement «ouverts d'esprit», comme nos interviewés aiment à le souligner - et le cadre du workshop sont les éléments qui engendrent l'échange interdisciplinaire.

Les éléments architecturaux pensés par les dirigeants du SFI semblent ainsi entretenir le travail interdisciplinaire plutôt que le créer. Ce qui ne veut pas dire que l'architecture du SFI n'a aucun rôle sur les échanges entre ses chercheurs. Par exemple, peu après la prise de possession du Cowan Campus par l'institut, l'archéologue George Gumerman déclare qu'au sein de ce dernier, les collaborations se produisent parce que l'on ne peut pas ne pas collaborer: "there's no place to hide! », dit-il de façon humoristique (SFI, 1995-1996, p. 13). Dans les témoignages des membres du SFI, l'accessibilité des bureaux et surtout la règle, non écrite mais largement diffusée, de la liberté d'interaction, facilitent le transfert de connaissances. En outre, il faut rappeler que le SFI est un visiting institute, les membres permanents n'étant qu'une dizaine. L'autre centaine de personnes se compose de chercheurs qui viennent une semaine ou un mois par an, ou bien à l'occasion d'une année sabbatique. Comme le remarque l'«éconophysicien» Blake LeBaron, l'état d'esprit dans lequel se trouvent les visiteurs de l'institut est fondamental pour la réussite de la collaboration interdisciplinaire ${ }^{8}$. Libérés de tous les devoirs qui leur incombent quotidiennement dans leur activité universitaire, ils sont prédisposés à l'interaction.

\section{Le cas d'étude de I'ISC-PIF}

Comme le SFI, l'ISC-PIF a changé de siège au cours de son histoire. "Dans les [premiers] locaux, explique son fondateur, il y a[vait] des salles de réunion. Il y a[vait] des bureaux mobiles : le matin, celui qui arriv[ait] pren[ait] la place ${ }^{9} »$. Le centre était situé au rez-dechaussée dans un immeuble moderne du 57-59 rue Lhomond, dans le $5^{\mathrm{e}}$ arrondissement de Paris et avait des bureaux au demi-niveau supérieur, ainsi qu'un espace de coworking au demi-niveau inférieur, que les membres de l'institut appelaient la "fosse ${ }^{10}$ ». Selon plusieurs personnes, cette fosse était l'élément ayant créé les liens entre les membres de l'institut. En outre, dans ce premier siège, les portes des bureaux restaient ouvertes, tout le monde pouvant entendre les autres parler, y compris pendant les conseils d'administration. Comme le

\footnotetext{
${ }^{8}$ Entretien avec Blake LeBaron, 14/09/2016.

${ }^{9}$ Entretien avec Paul Bourgine, 27/05/2013.

${ }^{10}$ Entretien avec Romain Reuillon, 31/01/2017.
}

SFI, l'ISC-PIF met l'accent sur le dynamisme, sur l'informalité, sur l'horizontalité des rapports qui y ont lieu: «il faut absolument que ça soit un lieu d'échanges, de croisements, d'interactions. Ça doit représenter un peu la science qu'il décrit ${ }^{11}$ ». L'ancêtre américain, le SFI, mais aussi les start-up de la Silicon Valley, en constituent les modèles.

Contraint pour des raisons financières de quitter les bureaux de la rue Lhomond, l'ISC-PIF, devenu une unité de service et de recherche du CNRS, obtient de la ville de Paris des locaux au 113 rue Nationale dans le $13^{\mathrm{e}}$ arrondissement.

\section{Les intérêts pluridisciplinaires des premiers membres}

Les fondateurs de l'ISC-PIF, ainsi que tous ses membres, sont friands de collaborations avec des spécialistes d'autres disciplines, ou bien se trouvent à cheval entre différents domaines du savoir. Prenons l'exemple de Paul Bourgine, premier directeur de l'ISCPIF et l'un des animateurs du RNSC : il est spécialiste à la fois de la modélisation numérique, des mathématiques, de l'économie, des sciences cognitives et de la philosophie. Le deuxième directeur du centre, Arnaud Banos, est un géographe qui maîtrise les statistiques et la modélisation informatique. Quant à l'actuel directeur, David Chavalarias, il est polytechnicien avec une spécialisation en informatique et des connaissances en sciences cognitives, économie et sociologie. Ces trois personnes sont assez représentatives des chercheurs gravitant autour de l'ISC-PIF.

\section{La planification de l'interdisciplinarité}

Les bâtiments des deux sièges de l'ISC-PIF n'ont pas pu être construits ex nihilo, comme ce fut partiellement le cas pour le dernier siège du SFI; en revanche la conception interne était sous la responsabilité de l'institut.

L'édifice qui héberge l'ISC-PIF aujourd'hui, appelé le Totem et accessible par deux entrées ${ }^{12}$, a été construit en 1997 par l'architecte Christian de Portzamparc. Il est conçu comme une «interface» pour encourager les échanges entre scientifiques, artistes et citoyens ${ }^{13}$. Lorsque l'un des organismes louant une partie de l'édifice l'a quitté, deux étages, puis trois, ainsi que l'auditorium sont devenus disponibles pour l'institut et c'est le directeur, David Chavalarias, qui a dessiné les plans de tous ces espaces. Lors de notre entretien, il a

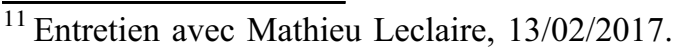

1211 place Nationale et 113 rue Nationale.

${ }^{13}$ Voir la page «Le Totem » sur le site de l'ISC-PIF : https:// iscpif.fr/projects/le-totem/.
} 
clairement exposé ses idées en matière de fonctionnalité et de symbolique attribuées au bâtiment.

Ainsi, au premier étage de l'édifice se trouve la salle des séminaires, avec une petite pièce rectangulaire attenante, utilisée pour les repas lors des journées d'études ou des conférences (Fig. 2).

Le deuxième étage contient l'open space dédié au coworking, ainsi que deux bureaux, un pour le directeur et un pour les assistantes gestionnaires (Fig. 2).

Le troisième étage contient deux bureaux qui hébergent temporairement des équipes affectées aux projets, puis une petite salle de réunion avec une bibliothèque et enfin une salle de pause d'accès libre et autoorganisée avec une cuisine, un hamac, une petite table, des plantes et des jeux de rôle (Fig. 2).

Le rez-de-chaussée n'a pas été utilisé de 2013 à 2017, faute de fonds pour le rénover. Aujourd'hui, il se compose de plusieurs salles, dont moins d'un quart appartient à l'ISC-PIF : il s'agit d'un autre espace de coworking. Les parties restantes sont partagées avec d'autres organismes.

Si l'objectif de Chavalarias est de faire circuler les chercheurs, le modèle reste celui de l' «incubateur» de start-up et du «tiers-lieu » (à mi-chemin entre la maison et le lieu de travail). La métaphore que le directeur emploie pour décrire la circulation des chercheurs et des usagers de son institut relève, par contre, du domaine de la physique : il explique avoir conçu la relation entre les différents étages de la structure à l'instar d'une «chambre à convection», dans laquelle les étages d'en bas sont le lieu où «ça bout ${ }^{14}$ ». Dans cette image, les activités des niveaux inférieurs sont censées créer des «moulins», soit des initiatives qui se consolident et montent étage par étage, jusqu'au dernier, celui de la «pépinière», où "ça se cristallise dans les bureaux un peu plus petits ${ }^{15} »$. La métaphore de la convection se couple à celle de la « distillation ${ }^{16} »$, car chaque étage que les personnes montent depuis le rez-de-chaussée jusqu'à la pépinière implique une augmentation de leur curiosité et de leur engagement.

D'autre part, l'ISC-PIF - sous l'égide de l'Institut des sciences humaines et sociales du CNRS qui est attentif à la problématique de la participation-se propose d'héberger des expériences de science citoyenne. La figure de l'incubateur vise donc non seulement à attirer des chercheurs, mais aussi des citoyens et des startupers. Pour ce faire, l'institut déploie plusieurs dispositifs, dont celui de journées portes

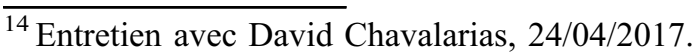

${ }^{15}$ Ibid.

${ }^{16}$ Ibid.
}
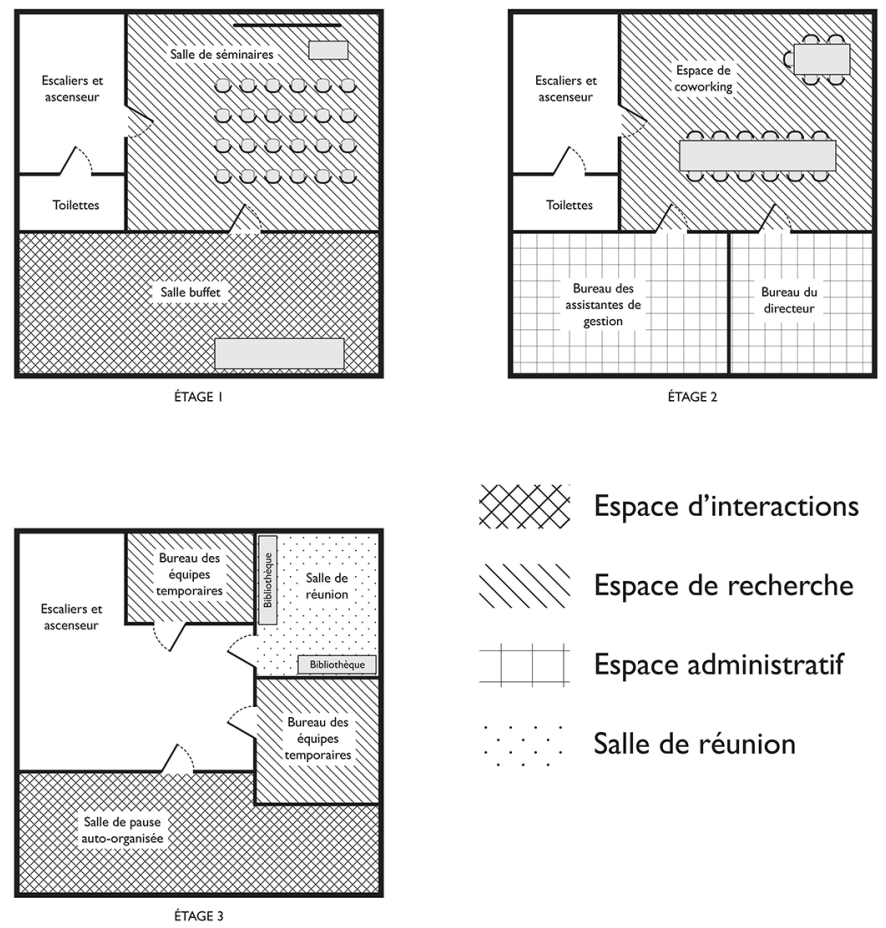

Espace d'interactions

Espace de recherche

Espace administratif

Salle de réunion

Fig. 2. Plans des trois étages du siège de l'ISC-PIF, 113 rue Nationale à Paris. Réalisés à partir des plans fournis par David Chavalarias, ces dessins en sont une version simplifiée et modifiée (crédit: Anne-Fleur de Sagazan).

ouvertes, occasion pour les scientifiques d'exposer leurs recherches à un large public.

\section{Où s'engendre l'interdisciplinarité ?}

Dans le cas de l'institut francilien, la phase de conception des collaborations scientifiques interdisciplinaires coïncide surtout avec l'écriture des projets aux financements européens, nationaux, régionaux ou privés. Une fois les fonds obtenus, les directeurs des projets embauchent des doctorants et des post-doctorants issus de plusieurs disciplines, et les mettent au travail en binôme ou par groupes. Les projets sont conçus dans des conditions les plus diverses, par exemple lors de dîners informels à la marge de conférences internationales, comme le témoigne une chercheuse en embryologie. Aujourd'hui, elle dirige l'unité BioEmergences au CNRS, dont les origines remontent au début des années 2000 quand, au sein de l'ISC-PIF, son équipe mène des recherches sur les embryons au moyen de microscopes laser achetés avec des financements européens :

«c'était 2002 et je vais à ce congrès, je rencontre Paul [Bourgine]. Et je me souviens d'un dîner de congrès [...] sous la coupole de l'un de ces grands magasins parisiens. [...] on s'est retrouvés assis côte à côte et on a discuté. [...] j'étais quasiment mûre pour monter ma propre équipe [...] on dit, on va faire des demandes de financement européen. 
[...] Et ça a été le fruit d'une réflexion où Paul me dit : "Si on devait faire un projet ensemble, il faudrait que ça soit quoi ? De quoi vous avez besoin?" [...] et moi je lui dis: "J'en peux plus d'être sur mon ordinateur avec un crayon à regarder les cellules à la main à tenter de me figurer où estce que la cellule que je vois au pas de temps t1 va se retrouver au pas de temps t2". Il me dit que ça peut s'automatiser et [on a monté] le tout premier consortium [de l'ISC-PIF] [réunissant biologistes et ingénieurs sous un même toit $]^{17}$ ».

D'après Chavalarias, l'ISC-PIF est conçu pour être un incubateur de nouveaux projets: les occasions de rencontre demeurent toutefois encadrées dans des dispositifs précis, tels que les journées portes ouvertes ou les conférences, et l'architecture a peu ou prou d'influence sur le processus. Une ex-doctorante de l'institut confirme en effet que les projets interdisciplinaires sont conçus soit en amont des financements, soit en dehors de l'institut dans des situations organisées ad hoc comme des excursions d'une semaine quelque part loin de Paris ${ }^{18}$.

L'espace de coworking est le lieu où se maintiennent ensuite les échanges autour de ces projets, conçus ailleurs. Lors de nos nombreuses visites à l'ISC-PIF, nous avons observé que, dans la salle de travail commune au deuxième étage, les personnes interagissent entre elles à voix basse de temps en temps et que quelques-uns travaillent avec un casque pour mieux se concentrer. Les interactions adviennent notamment lorsque certains ont des questions à poser à leurs collègues, que ce soit dans le cadre d'une recherche menée ensemble ou non. Si la discussion doit être approfondie, les interlocuteurs peuvent migrer ailleurs, par exemple dans la salle de réunion-bibliothèque, dans la salle de pause au troisième étage, ou ils peuvent reprendre leurs échanges au moment du déjeuner ou du goûter.

Les bureaux de l'open space ne sont pas mobiles, à la différence de la rue Lhomond, et ils sont individuels: chacun a son siège, les bureaux sont les uns à côté des autres, comme dans un centre d'appel (sans les cloisons entre les postes). Par rapport à la première période de l'ISC-PIF, les flux de personnes sont donc plus homogènes et classiques.

\section{Conclusion}

Les matériaux mobilisés ici ne permettent pas d'avancer de thèses définitives, ni sur les deux instituts de la complexité pris en exemple, ni sur l'architecture scientifique en général. Le but de ce texte a été de souligner que le lien entre l'architecture et les sciences est un objet intéressant d'enquête sociologique et de

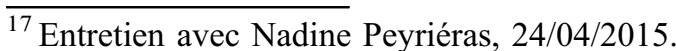

${ }^{18}$ Entretien avec Giulia Carra, 07/07/2018.
}

suggérer quelques points d'entrée pour l'étudier. Pour poursuivre les travaux commencés, il nous semblerait tout d'abord nécessaire d'analyser de près la dimension symbolique dans toute sa richesse: discours programmatiques, savoirs et pratiques, rapports de pouvoir, etc. (Delitz, 2018). En même temps, il faudrait également poursuivre les études comparatives et historiques commencées de manière un peu désordonnée par Galison et Thompson (1999), en poussant davantage l'apport des Science and Technology Studies (STS) dans l'étude des actions de l'architecture - un non-humain comme les autres (Yaneva et Guy, 2008). À cet égard, Müller et Reichmann (2015) proposent une palette d'instruments qualitatifs et dessinent un programme de recherche intéressant. Le premier consiste en trois méthodes d'enquête classiques, telles que l'observation, les entretiens et l'analyse documentaire, conçus de manière à saisir l'interrelation entre architecture et social. Quant au deuxième, il consiste précisément à rendre compte à la fois du niveau symbolique et du niveau matériel des objets architecturaux. Tout comme les STS qui permettent de dépasser la simple sphère symbolique, l'écueil à éviter, lorsqu'on adopte une approche matérialiste des technosciences, est de tomber dans la position qui attribue aux non-humains une causalité unidirectionnelle sur les pratiques sociales. En effet, déterminisme social et déterminisme technologique se repoussent autant qu'ils se complètent et se corrigent réciproquement. Un tel programme tient en trois questions fondamentales: comment les chercheurs conçoivent-ils et se réapproprient-ils l'architecture? Comment l'architecture cadre-t-elle et impacte-t-elle la vie des chercheurs? Et comment, en définitive, architecture et scientifiques coévoluent-ils ?

\section{Références}

Arthur W.B., Durlauf S.N., Lane D.A., 2015. Process and emergence in the economy, in Arthur W.B. (Ed.), Complexity and the economy, Oxford, Oxford University Press, 89-102.

Bourdieu P., 1980. Le mort saisit le vif. Les relations entre l'histoire réifiée et l'histoire incorporée, Actes de la recherche en sciences sociales, 32-33, 3-14, https://doi. org/10.3406/arss.1980.2077.

Bourgine P., Johnson J. (Eds), 2006. Living roadmap for complex systems science. Version 1.22, IST-FET Coordination Action, Open Network of Centres of Excellence in Complex Systems (ONCE-CS).

Bourgine P., Chavalarias D., Perrier E. (Eds), 2009. French roadmap for complex systems. 2008-2009 Edition, Paris, ISC-PIF, RNSC, IXXI, https://hal.archives-ouvertes.fr/hal00392486/en/.

Brand S., 1995. How buildings learn. What happens to them after they're built, Londres, Penguin. 
CNRS, INSERM, EP, ENS, UPMC, Paris 11, Paris 1, Institut Curie, Genopole, IHES, EHESS, ESPCI, ENS Cachan, Région Île-de-France, 2005. Convention constitutive du GIS «Institut des systèmes complexes Paris Ille-deFrance », Paris, Archives de l'ISC-PIF.

Cowan G., 2010. Manhattan Project to the Santa Fe Institute. The memoirs of George A. Cowan, Albuquerque, University of New Mexico Press.

Delitz H., 2018. Architectural modes of collective existence: architectural sociology as a comparative social theory, Cultural Sociology, 12, 1, 37-57, https://doi.org/10.1177/ 1749975517718435.

Galison P., 1997. Image and logic. A material culture of microphysics, Chicago, The University of Chicago Press.

Galison P., 1999. Buildings and the subject of science, in Galison P., Thompson E. (Eds), The architecture of science, Cambridge, MIT Press, 1-25.

Galison P., Thompson E. (Eds), 1999. The architecture of science, Cambridge, MIT Press.

Helmreich S., 1998. Silicon second nature. Constructing artificial life in a digital world, Berkeley \& Los Angeles, University of California Press.

Li Vigni F., 2018. Les systèmes complexes et la digitalisation des sciences. Histoire et sociologie des instituts de la complexité en France et aux États-Unis. Thèse de doctorat, Paris, École des hautes études en sciences sociales.

Müller A.-L., Reichmann W. (Eds), 2015. Architecture, materiality and society. Connecting sociology of architecture with science and technology studies, Hampshire, Palgrave.

Pines D. (Ed.), 1988. Emerging syntheses in science. Proceedings of the founding workshops of the Santa Fe Institute, Santa Fe, New Mexico. Volume I, New York, Perseus Books.

SFI, 1984. Discussion paper concerning the conception, creation and operation of the Rio Grande Institute, May 15, boîte d'archives «SFI early docs development archives ».
SFI, 1986. SFI mission statements, boîte d'archives « SFI concepts mission statements 1984-1989 ».

SFI, 1987. A proposal to develop a broad research program on the sciences of complexity at the Santa Fe Institute, Santa $\mathrm{Fe}$, New Mexico, August 24, boîte d'archives "SFI concepts mission statements 1984-1989 ».

SFI, 1988. The Bulletin of the Santa Fe Institute, WinterSpring, 3, 1.

SFI, 1989. The Bulletin of the Santa Fe Institute, Summer-Fall, $4,2$.

SFI, 1990. The Bulletin of the Santa Fe Institute, WinterSpring, $5,1$.

SFI, 1993. Facilities program. Santa Fe Institute. Prepared by Laban Wingert Associates, Santa Fe, New Mexico, November, boîte d'archives "Santa Fe Institute archival materials relating to acquisition of The Hyde Park Road Campus ».

SFI, 1995-1996. The Bulletin of the Santa Fe Institute, Winter, 11,1 .

SFI, 1996. The Bulletin of the Santa Fe Institute, Summer, 11, 2.

SFI, 1996-1997. The Bulletin of the Santa Fe Institute, Winter, $12,1$.

SFI, 2004. The Bulletin of the Santa Fe Institute, Fall, 19, 2.

Waldrop M.M., 1992. Complexity. The emerging science at the edge of order and chaos, New York, Simon \& Schuster Paperbacks.

Winner L., 1980. Do artifacts have politics?, Daedalus, 109, 1, 121-136, https://www.jstor.org/stable/20024652.

Wyatt S., 2008. Technological determinism is dead; long live technological determinism, in Hackett E.J., Amsterdamska O., Lynch M., Wajcman J. (Eds), The handbook of science and technology studies, Cambridge \& London, MIT Press, 165-180.

Yaneva A., Guy S., 2008. Understanding architecture, accounting society, Science and Technology Studies, 21, 1, 3-7, https://doi.org/10.23987/sts.55230.

Citation de l'article : Li Vigni F. Architecture et interdisciplinarité. Le cas d'étude des instituts de la complexité. Nat. Sci. Soc. 28, 3-4, 306-313. 